
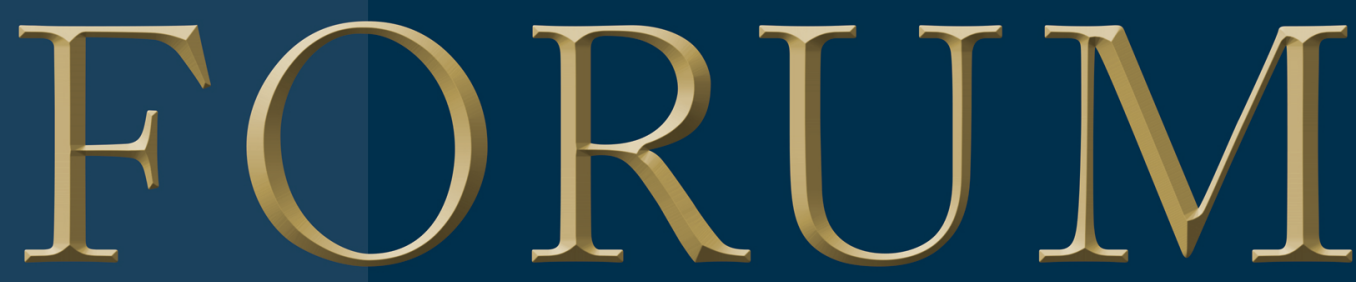

VOLUME 31 | NUMBER 4 JULY/AUGUST

HAIR TRANSPLANT FORUM INTERNATIONAL

IN THIS ISSUE

Case Study of Hair Regrowth with Topical Cannabidiol (CBD)

Preview Long Hair Follicular Unit Excision

Fenugreek and Management of Androgenetic Alopecia: Fact or Fiction

PLAN TO ATTEND!

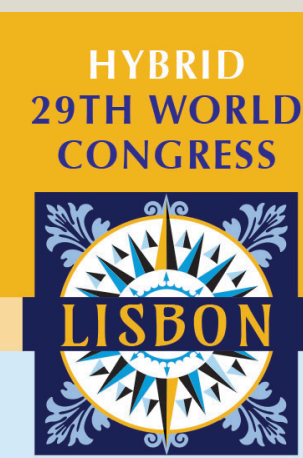

October 21-23, 2021

29thannual.org

Don't Miss

The Big One!

INTERNATIONAL SOCIETY OF HAIR RESTORATION SURGERY

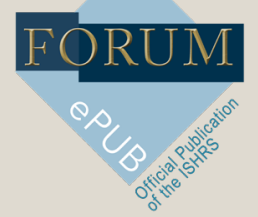

\section{FUE Heresy: The True Impact of FUE Follicle Transections on Follicle Survival and Hair Diameter Index Values}

\author{
James A. Harris, MD, FISHRS I Greenwood Village, Colorado, USA I jharris@hsccolorado.com
}

\section{ABSTRACT}

Introduction: Transected follicles have shown some ability to regenerate growing hair at a given rate depending on the location of the transection. To date, there has been no examination of the true impact of follicle transection rates given this follicle regeneration. This study attempts to quantify the impact of transections on the actual number of surviving follicles and determine the impact on the cosmetic outcome using the Hair Diameter Index (HDI).

Method: Follicle transection samples from 29 male patients undergoing FUE were evaluated for the level of transection and the location noted.

Results: There were 1,682 transected follicles examined. Approximately 45\% of transections were in the middle third and the remainder were split evenly in the upper and lower thirds.

Conclusion: Based on the studies showing follicle regeneration, it is estimated that 953, or 57\%, of the 1,682 transected follicles would be expected to survive. With the survival of transected follicles, the visual impact of transections may be minimal. Using the equation for the HDI calculation, the result suggests that a transection rate of $32 \%$ would only start to make a significant visual difference in the transplant density. It is apparent that transections should be minimized, but great efforts should be made to prevent factors such as desiccation and handling trauma, which can harm a significant number of follicles, as these factors may have a greater role in the cosmetic outcome of an FUE procedure.

Key Words: follicle regrowth, FUE, Hair Diameter Index (HDI), hair density, transection

This article is open access and may not be copied, distributed, or modified without written permission from the International Society of Hair Restoration Surgery.

\section{INTRODUCTION}

It has been well demonstrated that transected follicles have the ability to regenerate growing hair, ${ }^{2-4}$ however, the extent to which this is possible depends on the location of the follicle transection. Kim's work indicates that the survival rates for follicle portions, where the follicle was transected at various levels, are as follows:

Upper third (lower two-thirds intact planted) $-83 \%$

Lower third (upper two-thirds intact planted) $-65 \%$

Middle third (upper half planted) $-40 \%$

Middle third (lower half planted) $-27 \%$

Current FUE devices have made FUE fast and efficient with transection rates in the 2-5\% range possible. In my own experience, I have found that as the speed of FUE increases and the punch cutting diameter decreases, the rate of follicle transection increases.

There seems to be an "inviolable" rule that any follicle transection results in irreparable harm and all transections should be avoided. Based on the works cited above, that maxim may not be entirely grounded in fact. To date, there has been no examination about the true impact of a marginal increase in follicle transection rates given the follicle regeneration rates in the studies noted above.

Some of the conclusions from this study are sure to be controversial as they contradict long-held principles. 


\section{OBJECTIVE}

This study attempts to quantify the potential impact of follicle transections in different follicle locations on the actual number of surviving follicles and determine the impact on the cosmetic outcome using the Hair Diameter Index (HDI).

\section{MATERIALS AND METHODS}

Follicle transection samples from 29 male patients undergoing FUE harvests using the SAFE System with a $0.8 \mathrm{~mm}$ blunt punch were obtained. The follicles were evaluated for the level of transection and noted whether it was in the upper third, middle third, or lower third. In the cases of the middle third transections, it was also noted whether it was the upper or lower half of the follicle that remained with the graft. This is important as the implantation of upper or lower half results in a different survival rate as noted in Kim's work. In the cases where there was an upper third or lower third transection, the intact two-third's portion remained with the graft.

\section{RESULTS}

The case sizes ranged from 145 grafts to over 3,200 grafts with the case follicle transection rate ranging from $2.8 \%$ to 8.4\%. Approximately 50-65 random transection samples from each case were evaluated.

There were 1,682 transected follicles examined and they were categorized as follows:

Upper third - $462(27.5 \%)$

Lower third - $468(27.8 \%)$

Middle third - $752(44.7 \%)$

Upper half follicle planted - 579

Lower half follicle planted - 173

Given the survival rates noted by Kim, the expected follicle survival of the transected follicles in the recipient area are as follows:

Upper third $-462 \times .83=383$

Lower third $-468 \times .65=304$

Middle third - 752

Upper half follicle intact $-579 \times .40=232$

Lower half follicle intact $-173 \times .27=47$

966 , or $57 \%$, of transected follicles expected to regenerate

\section{DISCUSSION}

Based on the work by Kim, there is an expected rate of follicle regeneration after transection depending on the level of transection. In this study, there were 1,682 follicles transected, and assuming Kim's results are generalizable, in the recipient area, we could expect 966 of the 1,682 transected follicles (57\%) to result in a regenerated follicle. The effective rate of follicle loss in the recipient area due to transections would be $43 \%$, or 723 follicles. As an example, if an average follicle transection rate for a case is approximately 5\%, we would expect that only 2-3 hairs/100 hairs extracted would suffer a transection and not survive. Even at a $10 \%$ transection rate, that is $4-5$ hairs out of 100 hairs extracted lost due to transection. This alone would not result in a significant loss of follicles.
Most of the follicle transections occurred in the middle third of the follicle. In analyzing the graft and the damaged follicles, it appeared that the majority of these transections may have been prevented by more accurate centering of the target follicular unit as the transected follicles appeared to be the follicles on the periphery of the graft. The transections in the upper third seemed to be related to either centering issues or inaccurate estimation of the emergence angle of the graft. These errors may have been mitigated by either a more accurate placement of the punch, that is, a slower rate of dissection, or by using a larger diameter punch. The distal transections would likely have been prevented by depth limitation or a larger punch.

The nuance in the significance of the mid-third transection is that both the upper and lower portion have the possibility of regeneration. If we consider the 752 follicles that were transected in this region, and using the regeneration values by Kim, 225 follicles, in addition to the ones regenerating in the recipient area, would have been expected to survive in the donor region. This means that 504 of the 753 follicles transected in the middle third, or $67 \%$, will regenerate either in the donor or recipient areas.

Another point to consider is whether to trim follicle fragments when present on the graft before implantation. If the rule is that one should trim follicle fragments when less than two-thirds of the follicle is present, this would have resulted in 279 fewer follicles regenerating in the patient's recipient areas.

The impact of the transections on scalp coverage can be assessed using the equations to calculate the HDI.5 An HDI of 60 or greater is associated with an adequate hair density for good scalp coverage. To be conservative, if we assume that a 10-point decrease in the HDI is enough to detect a visual change the hair density, we can solve the equation to calculate the transection rate that would result in a 10-point drop in the HDI and represent a significant difference in visual density.

If one were to create a transplant density HDI of 60 , and we assume a hair caliber of 70 microns, the number of hairs required is 85 hairs $/ \mathrm{cm}^{2}$ (approximately 40 follicular units/ $\mathrm{cm}^{2}$ ). To achieve a visible decrease in density to an HDI of 50 , the ending hair count $/ \mathrm{cm}^{2}$ has to be 71 . The average follicle death rate (no regeneration) due to transection, after taking into account the various levels of transections, is $43 \%$. If we transected 32 hairs out of the 85 (37\% transection rate), and assumed a $57 \%$ regeneration of the transected hairs, this would yield a final hair density of $71 / \mathrm{cm}^{2}$ and an HDI of 50 .

Put simply, in this scenario, a transection rate of $37 \%$ would only start to make a significant difference in the transplant density, that is, lowering the HDI from a value of 60 to 50 . I am not suggesting that we can be lackadaisical about minimizing transection rates, but it requires significant transection rates to cause a significant degradation in visual density.

There are several noteworthy points that should be made about the shortcomings of the study. The reader should be aware of the following:

1. There are possible inaccuracies in the survival rates published by Kim.

2. Percentages of the levels of transections differs from practice to practice. 
3. The level of transection is subject to interpretation.

4. The level of transection is not a discrete variable, but rather it is continuous.

5. The overall follicle survival rate for any FUE transplant procedure is unknown.

6. There is evidence that follicle regeneration is associated with a diminished hair shaft diameter which is not accounted for.

\section{CONCLUSION}

Based on the study by Kim that suggested that transected follicles survive or regenerate to a certain extent depending on the location of transection, a significant rate of follicle transection may be needed before there is a meaningful impact on the visual transplant density. Of course, follicles are a non-renewable resource and every attempt should be made to ensure a "reasonable" case transection rate. Transections should be monitored in every case and appropriate adjustments made.

If we examine a case of maximal grafts extracted by FUEfor example, 12,600 follicles (approximately 6,000 grafts) with a $10 \%$ follicle transection rate, and there is a $43 \%$ follicle demise rate due to transection in the transplanted area, there would be 542 follicles (approximately 258 follicular units) that are irrevocably lost. If these non-surviving follicles were spread over the 200 square centimeters of the frontal and mid-scalp transplanted regions, this loss would have been 1-2 follicles $/ \mathrm{cm}^{2}$, which represents an inconsequential difference.

A surgeon must be aware of the many factors that can impact the follicle transection rate, such as rate of excision, punch size, etc., and balance these factors against out-ofbody time, methods of implantation, and surgical goals. The impact of transection rates under $10 \%$ are likely miniscule. Factors that impact a larger proportion of the follicles, such as desiccation, handling, storage solutions, and implantation techniques, likely have a much larger impact on graft survival than a nominal increase in follicle transection rate. It is my suggestion that greater care and attention be paid to those factors that potentially cause the failure of the majority of grafts rather than spending in inordinate amount of time and effort in technique and instrument changes for a small improvement in the follicle transection rate.

\section{References}

1. Harris J. Poster presentation: FUE Evaluation of Follicle Transection Locations and Its True Impact on Follicle Survival and Hair Diameter Index (HDI) Values. 28th Annual Meeting of the ISHRS, Virtual; October 2020.

2. Mayer MA. Follicular Regeneration. Presented at the 6th Annual Meeting of the ISHRS, Washington, DC; September 1998.

3. Kim JC, Choi YC. Regrowth of grafted human scalp hair after removal of the bulb. Dermatol Surg. 1995; 21:312-313.

4. Devroye J. FUE, regrowth rate of transected hair in the donor area. Hair Transp Forum Int'l. 2015; 25(6):238-239.

5. Harris J. Poster presentation: Application of the Hair Diameter Index (HDI) in Obtaining a Target Hair Density. Presented at the 11th Annual Meeting of the ISHRS, New York, New York; October 2003.

\section{Invited Commentary}

\section{David Josephitis, DO, FISHRS I Bloomington, Minnesota, USA}

With "FUE Heresy" in the title, this article is sure to raise eyebrows. I am glad this study was performed as it really helps to shed some light on the true cosmetic effect of follicular transection rate (FTR) during FUE, and how we should put it into perspective. We have known for years that transected grafts harvested via FUE are not always completely lost. Kim's work as referenced here gives us a hopeful estimate that a good number of transected hairs can still grow (57\% using the data from this study by Harris)., ${ }^{1,2}$ At the time of publication, these studies were well received and were helpful for many surgeons trying to understand the importance of minimizing transection in their patients. That was then though, and this is now. This study shows how the impact of these transections today may not be as "cosmetically" important as we once believed them to be.

Much has changed in a short period of time since the advent of these original studies. As mentioned in the article, devices have improved dramatically. Also, the experience level of the surgeons has greatly increased. With the early use of manual and other sharp punches, only a few mastered the skills necessary to maintain low FTRs in most of their patients. For many other practitioners new to the technique of FUE, the learning curve was quite steep and poor growth of grafts with higher FTR was more common. Surgeons years ago were very keen to focus their attention on lowering the FTR to save donor and improve the overall viability of the transplanted grafts. Within the past decade though, the overall FTR in experienced hands has decreased. Also, the modern devices available have helped make lower FTR a reality for more physicians. This, in addition to the overall care of the grafts after extraction, (e.g., implanters and holding solutions) has produced the high quality of FUE results seen today.

It was interesting to see that with an average case $(6,000$ FUs, $10 \%$ FTR, and $43 \%$ demise rate of transected follicles), only 542 follicles ( $258 \mathrm{FUs}$ ) were lost. These lost follicles are also, most likely only a portion of a larger 2- or 3-hair graft, thus making the cosmetic difference even smaller. Although we have been trained to hold all follicular life sacred, this is not a large number of follicles. Having said that, we should still strive to keep the FTR low but, at the same time, keep the actual loss of grafts in perspective.

Now, there are some caveats that should be considered. If different devices are used, less-experienced hands are employed, and/or more difficult cases are encountered, the number of lost follicles will undoubtably increase. ${ }^{3,4}$ Also, if alternative devices other than those in this study are used, the percentage of transections at various levels may change, affecting the overall rate of transected graft death. Another point to ponder is the common practice of harvesting outside of the traditional Safe Donor Area (SDA). Large numbers of grafts are oftentimes removed from both above and below areas the SDA for multiple reasons (e.g., large session, repeat session, or homogenization of donor area). True discussed this in his most recent article in this publication, 